\section{Perte d'inhibition \\ neuronale et spasticité \\ après traumatisme de la moelle épinière}

Pascale Boulenguez, Sylvie Liabeuf, Laurent Vinay

\author{
Laboratoire plasticité et physiopathologie de la motricité \\ (UMR6196, CNRS et Aix-Marseille Université), \\ 31, chemin Joseph Aiguier, \\ 13402 Marseille Cedex 20, France. \\ vinay@dpm.cnrs-mrs.fr
}

> Le bon fonctionnement du cerveau et de la moelle épinière requiert un équilibre entre l'activité des neurones excitateurs et celle des neurones inhibiteurs. À titre d'exemple, l'alternance entre la contraction des muscles extenseurs et fléchisseurs de la jambe au cours de la marche est permise par la mise en jeu de neurones inhibiteurs situés dans la moelle épinière lombaire. Après une lésion de la moelle épinière, l'altération des processus inhibiteurs [1] conduit à l'hyperactivité de certains réflexes, une raideur musculaire, une co-contraction des muscles antagonistes et des spasmes douloureux, qui caractérisent la spasticité. Cette pathologie apparaît chez $75 \%$ des patients paraplégiques et tétraplégiques et peut altérer très profondément leur qualité de vie. Nous avons identifié récemment les mécanismes responsables de cette altération de l'inhibition neuronale [2].

\section{L'effet inhibiteur \\ des neurotransmetteurs \\ est sous le contrôle}

de la concentration en ions chlorure

Les principaux neurotransmetteurs inhibiteurs dans la moelle épinière sont l'acide $\gamma$-aminobutyrique (GABA) et la glycine. Les récepteurs de la glycine et un certain type de récepteurs du GABA (les récepteurs $G A B A_{A}$ ) sont associés à des canaux ioniques perméables aux ions chlorure $\left(\mathrm{Cl}^{-}\right)$[3]. Lorsque la concentration en ions chlorure $\left(\left[\mathrm{Cl}^{-}\right]_{\mathrm{i}}\right)$ dans les cellules cibles est faible, l'effet du GABA et de la glycine est de type inhibiteur (Figure 1).
L'ouverture des canaux provoque en effet dans ce cas une entrée de $\mathrm{Cl}^{-}$et donc une hyperpolarisation du potentiel de membrane du neurone qui s'éloigne du seuil de déclenchement des potentiels d'action indispensables à la propagation de l'influx nerveux. L'effet du GABA et de la glycine peut s'inverser lorsque la concentration en ions chlorure est forte, comme c'est le cas très tôt au cours du développement [4]. La $\left[\mathrm{Cl}^{-}\right]_{i}$ est régulée par des transporteurs d'ions situés dans la membrane des neurones. Ces protéines transportent à la fois des cations - sodium $\left(\mathrm{Na}^{+}\right)$ou potassium $\left(\mathrm{K}^{+}\right)$- et des $\mathrm{Cl}^{-}$. Certains de ces transporteurs font entrer le $\mathrm{Cl}^{-}$, par exemple les transporteurs $\mathrm{Na}^{+}, \mathrm{K}^{+}$et $\mathrm{Cl}^{-}$de type $\mathrm{NKCCl}$, d'autres transportent les ions $\mathrm{Cl}^{-}$hors de la cellule, les transporteurs $\mathrm{K}^{+}$et $\mathrm{Cl}^{-}$de type $\mathrm{KCC2}$. L'expression des $\mathrm{NKCCl}$ est particulièrement élevée dans les neurones immatures [3]. Le développement des KCC2 est responsable de la réduction de la $\left[\mathrm{Cl}^{-}\right]_{\mathrm{i}}$ au cours de l'ontogenèse et permet donc la maturation de l'inhibition post-synaptique $[3,5,6]$. Des travaux récents réalisés au niveau de plusieurs régions du système nerveux central ont démontré que l'expression des transporteurs KCC2 était réduite dans des conditions pathologiques (épilepsie, douleurs chroniques induites par une lésion de nerf périphérique [7]).

\section{Réduction de l'expression des KCC2 après lésion de la moelle épinière}

Comme nous l'avons déjà mentionné, la spasticité est liée à un dysfonction- nement des processus inhibiteurs, et son origine n'était jusqu'à ce jour pas connue. Nous avons testé les effets d'une lésion de la moelle épinière au niveau thoracique chez le rat (section totale ou contusion modérée, cette dernière étant plus représentative des traumatismes médullaires observés chez l'homme) sur I'expression des cotransporteurs KCC2 dans le tissu nerveux sous-lésionnel. Nous avons montré par des techniques classiques (Western blots) que l'expression des KCC2 commence à chuter dès le premier jour suivant la lésion de la moelle épinière et reste basse pendant les mois qui suivent, lorsque la spasticité se développe. L'analyse en microscopie confocale du marquage immunohistochimique des KCC2 a révélé une diminution de la densité de ces transporteurs au niveau de la membrane plasmique des neurones moteurs spinaux (motoneurones) chez les animaux lésés (Figure l). La quantité de KCC2 est en revanche accrue dans le cytoplasme suggérant que la translocation de la protéine vers la membrane plasmique ou sa stabilité au sein de cette membrane sont affectées par le traumatisme.

\section{Le blocage pharmacologique des KCC2} chez l'animal intact mime la spasticité Si la réduction d'expression des KCC2 provoquée par la lésion de la moelle épinière est en partie responsable de la spasticité, alors un blocage de ces transporteurs chez l'animal intact devrait avoir des conséquences fonctionnelles 


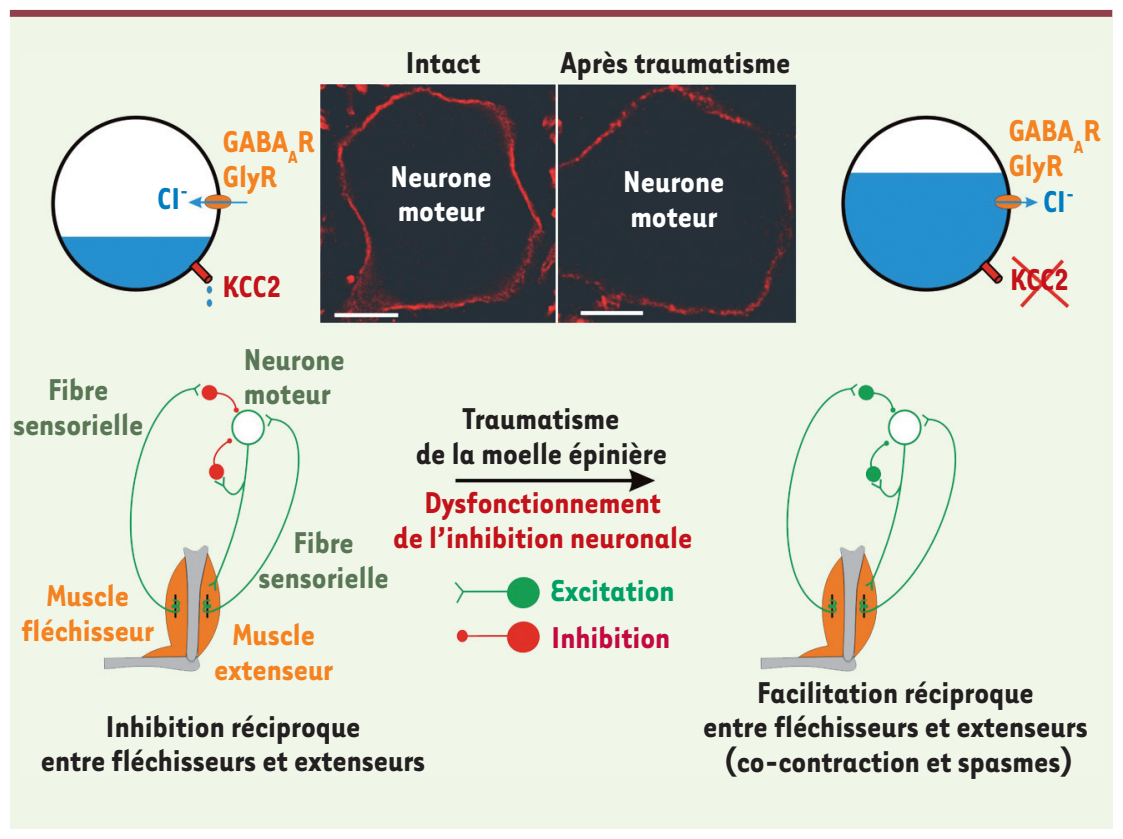

similaires. Afin de tester cette hypothèse, nous avons réalisé des injections intrathécales de DIOA ([ (dihydroindenyl) oxy]alkanoic acid), afin de bloquer les KCC2 au niveau de la moelle lombaire. Nous avons évalué les effets de cette injection sur le réflexe de Hoffmann (réflexe $\mathrm{H}$ ) du muscle extenseur de la cheville, qui consiste en une contraction du muscle en réponse à la stimulation électrique des fibres sensorielles. L'utilisation de stimulations répétées à hautes fréquences $(0,5 \mathrm{à} 5 \mathrm{~Hz})$ provoque normalement une dépression de plus en plus importante de l'amplitude de ce réflexe. Cette dépression est moins prononcée chez le patient paraplégique, et il s'agit là d'un corrélat fiable du développement de la spasticité après lésion de la moelle épinière [8]. Conformément à notre hypothèse, nous avons observé que l'injection de DIOA chez le rat intact réduit, de manière réversible, la dépression du réflexe $\mathrm{H}$ en réponse aux stimulations à hautes fréquences. Nous avons également utilisé des souris dont l'expression de KCC2 est réduite génétiquement de $50 \%$ [9]. Chez ces souris transgéniques, l'accentuation de la dépression du réflexe $\mathrm{H}$ en réponse aux stimulations répétées à hautes fréquences était plus faible que celle observée chez des souris contrôles. Ces résultats confirment qu'une chute d'expression de KCC2 après lésion de la moelle épinière peut rendre compte de l'hyperréflexie qui est associée à la spasticité.

\section{Comment sauver}

\section{les transporteurs KCC2}

après lésion de la moelle épinière?

Le BDNF (brain-derived neurotrophic factor), qui se fixe aux récepteurs TrkB (neurotrophic tyrosine kinase receptor type 2), est un facteur essentiel pour la survie des neurones. Ce facteur a un effet contrasté au cours du développement : il augmente l'expression de KCC2 au niveau de cellules immatures, mais la diminue dans ces mêmes cellules parvenues à un stade mature. L'application de BDNF sur des neurones corticaux adultes réduit ainsi l'expression de KCC2 [10]. Nous avons confirmé ces résultats au niveau des motoneurones spinaux en réalisant une injection intrathécale de BDNF chez le rat intact. De plus, la dépression du réflexe $H$ pour des stimulations à hautes fréquences était réduite parallèlement à la diminution des KCC2 dans la membrane plasmique des motoneurones. Le blocage de la liaison du BDNF à
Figure 1. L'activité des neurones moteurs est régulée par des circuits réflexes. Ces circuits proviennent de la périphérie sensorielle, les uns excitateurs (en vert), les autres inhibiteurs (en rouge). Un neurone est inhibé par l'activation des récepteurs $(R) G_{A B}$ et glycine si sa concentration en ions chlorure est maintenue à un taux faible par des transporteurs KCC2 dans la membrane qui expulsent ces ions. La photographie de gauche montre le marquage immunohistochimique de ces transporteurs au niveau d'un neurone moteur spinal chez un rat adulte intact. Le marquage consiste en une bande homogène tout au long de la membrane plasmique. En revanche, après une lésion de la moelle épinière, ce marquage devient moins intense et discontinu, comme le montre la photographie de droite. II résulte de cette diminution d'expression des KCC2 une augmentation de la concentration intracellulaire en chlorure et les neurones inhibiteurs deviennent excitateurs.

ses récepteurs TrkB concomitamment à la lésion de la moelle épinière (donc à un stade où les neurones sont encore considérés comme sains) a entraîné 24 heures après une augmentation de la quantité (donc une certaine sauvegarde) de KCC2 comparativement aux animaux lésés non traités. Ceci suggère que le BDNF pourrait être en partie responsable de la chute précoce de KCC2 après une lésion de la moelle épinière.

En revanche, on sait que le BDNF augmente KCC2 après axotomie, comme il le fait à des stades développementaux précoces [11]. De fait, lorsque nous avons réalisé des injections intrathécales de BDNF quinze jours après la lésion de la moelle, nous avons observé, 24 heures après l'injection, une augmentation de la quantité de KCC2 et de la dépression du réflexe $H$ pour des stimulations à hautes fréquences, ces deux paramètres se rapprochant ainsi des niveaux observés chez les animaux intacts.

\section{En conclusion}

Cette étude ouvre des perspectives novatrices pour le traitement de la spasticité face à laquelle les médecins sont actuellement relativement démunis. De nouvelles stratégies ciblant les systèmes de régulation du chlorure intracellulaire devraient permettre de restaurer l'inhibi- 
tion neuronale dans la moelle épinière et pourraient être utiles dans d'autres pathologies telles que la douleur neuropathique $[12,13] . \diamond$

Reduced neuronal inhibition and spasticity following spinal cord injury

\section{REMERCIEMENTS}

Ce travail a été soutenu par la Christopher and Dana Reeve Foundation et l'Institut pour la recherche sur la moelle épinière et l'encéphale (IRME).

\section{CONFLIT D'INTÉRÊTS}

Les auteurs déclarent n'avoir aucun conflit d'intérêts concernant les données publiées dans cet article.

\section{RÉFÉRENCES}

1. Boulenguez P, Vinay L. Strategies to restore motor functions after spinal cord injury. Curr Opin Neurobiol 2009; 19: 587-600.

2. Boulenguez $P$, Liabeuf $S$, Bos $R$, et al. Down-regulation of the potassium-chloride cotransporter KCC2 contributes to spasticity after spinal cord injury. Nat Med $2010 ; 16: 302-7$.

3. Ben-Ari Y. Le GABA : un transmetteur pionnier pour la construction du cerveau. Med Sci (Paris) 2007 ; $23: 751-5$.

4. Jean-Xavier C, Mentis GZ, O'Donovan M, et al. Dual personality of GABA/glycine-mediated depolarizations in the immature spinal cord. Proc Natl Acad Sci USA 2007 ; 104 : 11477-82.

5. Jean-Xavier C, Pflieger J-F, Liabeuf S, Vinay L. Inhibitory post-synaptic potentials in lumbar motoneurons remain depolarizing after neonata spinal cord transection in the rat. J Neurophysiol $2006 ; 96: 2274-81$.

6. Stil A, Liabeuf S, Jean-Xavier C, et al. Developmental up-regulation of the potassium-chloride cotransporter type 2 in the rat lumbar spinal cord. Neuroscience 2009 ; 164 : 809-21.
7. Kahle KT, Staley KJ, Nahed BV, et al. Roles of the cation-chloride cotransporters in neurological disease. Nat Clin Pract Neurol $2008 ; 4$ : 490-503.

8. Grey MJ, Klinge K, Crone C, et al. Post-activation depression of Soleus stretch reflexes in healthy and spastic humans. Exp Brain Res 2008 ; 185 : 189-97.

9. Woo NS, Lu J, England R, et al. Hyperexcitability and epilepsy associated with disruption of the mouse neuronal-specific $\mathrm{K}-\mathrm{Cl}$ cotransporter gene. Hippocampus 2002 ; 12 : 258-68.

10. Rivera C, Li H, Thomas-Crusells J, et al. BDNFinduced TrkB activation down-regulates the $\mathrm{K}^{+}-\mathrm{Cl}^{-}$cotransporter $\mathrm{KCC} 2$ and impairs neuronal $\mathrm{Cl}^{-}$ extrusion. J Cell Biol 2002 ; 159 : 747-52.

11. Shulga A, Thomas-Crusells J, Sigl T, et al. Posttraumatic GABA(A)-mediated $\left[\mathrm{Ca}^{2+}\right]$ i increase is essential for the induction of brain-derived neurotrophic factor-dependent survival of mature central neurons. J Neurosci 2008; 28 : 6996-7005.

12. Coull JA, Boudreau D, Bachand K, et al. Trans-synaptic shift in anion gradient in spinal lamina I neurons as a mechanism of neuropathic pain. Nature 2003; 424 : 938-42.

13. Ruggiero F. Les canalopathies de la douleur. Med Sci (Paris) $2010 ; 26: 1015-7$.
NOUVELLE

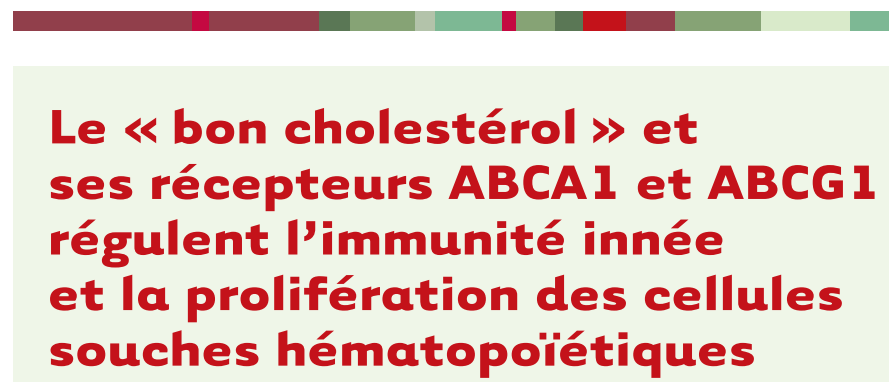

Emmanuel L. Gautier, Laurent Yvan-Charvet

\section{L. Gautier : Department}

of gene and cell medicine, Mount Sinai School of Medicine, New York, Ny 10029, États-Unis.

L. Yvan-Charvet:

Division of molecular medicine,

Department of medicine,

Columbia University,

630 West $168^{\text {th }}$ Street,

New York, Ny 10032, États-Unis.

ly2159@columbia.edu

développement d'une plaque d'athérome composée essentiellement de macrophages inflammatoires gorgés de lipides [2]. La mort de ces macrophages, que provoque la mauvaise élimination de ces lipides, conduit à la formation de débris cellulaires et entretient l'inflammation au sein de la plaque, favorisant ainsi sa progression [3, 4]. La présence des macrophages dans la plaque athéromateuse est consécutive au recrutement de monocytes circulants [5] et la persistance de ces macrophages dans la plaque pourrait être favorisée dans un contexte d'hyperlipémie [6] (Figure 1). Les HDL, en favorisant l'efflux du cholestérol des macrophages spumeux via ses transporteurs $A B C A l$ et $A B C G l$, diminuent le statut inflammatoire des et biochimique de ces lipides induit une réaction inflammatoire qui favorise le

\section{l'immunité innée dans le macrophage}

L'athérosclérose est caractérisée par la formation d'une plaque d'athérome en réponse au dépôt de lipides, en particulier de cholestérol, dans la paroi murins de la maladie. Nous avons précéces le rôle des transporteurs $A B C A l$ et $A B C G l$ dans l'efflux de cholestérol induit de nouveaux mécanismes athéroprotecteurs des HDL et de ses transporteurs

\begin{abstract}
été mis en évidence dans des modèles
de transport inverse du cholestérol. Le transporteurs de type $A B C$ (ATP binding «bon cholestérol ») et la diminution du risque cardiovasculaire. Le rôle proteccité à favoriser l'efflux du cholestérol des tissus périphériques vers le foie, et donc son excrétion via la voie dite rôle athéroprotecteur des HDL et de ses

> L'athérosclérose est la cause majeure
des maladies cardiovasculaires et est à l'origine d'environ $50 \%$ des décès dans les pays industrialisés. De nombreuune corrélation positive entre le taux plasmatique de lipoprotéines de haute
\end{abstract}

